\title{
An Assessment of Renal Function with Pulsatile Perfusion During Proximal Graft Using Cardiac Contraction in Coronary Artery Bypass Graft Surgery
}

\author{
Seyed Mohammadreza Amouzegar, ${ }^{1}$ and Marzieh Lak ${ }^{1, *}$ \\ ${ }^{1}$ Department of Anesthesiology, School of Medical Sciences, Baqiyatallah University of Medical Sciences, Tehran, Iran \\ "Corresponding author: Marzieh Lak, Baqiyatallah University of Medical Sciences, Tehran, Iran. Tel: +98-9125262585, Fax: +98-2188053766, E-mail: marziehlak@yahoo.com
}

Received 2017 April 17; Revised 2017 May 31; Accepted 2017 August 20.

\begin{abstract}
Background: Short-term pulsatile perfusion during cardio pulmonary bypass has shown higher protection for renal physiology, following standard preservation of glomerular filtration and reduction of renal tissue damage.

Methods: In this randomized clinical trial study, 70 patients, who had undergone CABG surgery were divided to 2 groups; pulsatile and non-pulsatile groups. The researchers transferred continuous blood flow to pulsatile blood flow during proximal graft using cardiac contraction. Patients' laboratory tests, such as blood urea nitrogen (BUN) and creatinine were checked preoperatively, at the arrival of the open heart intensive care unit (ICU-OH), and 24 and 48 hours after admission.

Results: Both case and control groups had significantly increased BUN and creatinine. Increased creatinine on the first and second postoperative day was significantly higher in the control group. Furthermore, BUN increased on the second day in the control group and was significantly higher than the case group

Conclusions: Despite the rise in BUN and creatinine in the 2 groups, there were no cases of renal failure in the patients. However, the results of this study regarding creatinine and BUN criteria were supported by the pulsatile perfusion method during the proximal graft by using a cardiac contraction in CABG surgery.
\end{abstract}

Keywords: Pulsatile Perfusion, Cardio Pulmonary Bypass, Renal Function

\section{Background}

Short-term pulsatile perfusion compared to nonpulsatile in elderly patients has shown higher protection for renal physiology during cardio pulmonary bypass (CPB), following standard preservation of glomerular filtration and reduction in renal tissue damage (1).

Pulsatile blood flow is a simple and harmless method preventing renal injury in elderly patients (2).

Compared to nonpulsatile blood flow, pulsatile blood flow is closer to normal physiology and results in improvement of micro-circulation as well as lowering systemic vascular resistance (3).

Nonpulsatile CPB causes metabolic acidosis and tissue oxygen consumption reduction, while pulsatile flow during CPB causes higher oxygen consumption and metabolic acidosis reduces (4).

Pulsatile CBP maintained renal function, decreased liver injury, decreased levels of endotoxins, and led to smaller demand for inotropic agents, and a lowering number of death postoperatively (5).

Accordingly, the purpose of this clinical trial study was to design an assessment of the effect of pulsatile blood flow in renal function during proximal graft in coronary artery bypass graft (CABG) surgery.

\section{Methods}

This study was a randomized clinical trial with RCT number IRCT2016102230421N1, which was composed of 72 consecutive patients, who underwent CABG surgery. Ethics committee approval was received along with informed consent of each patient. The same surgical team operated on all of the patients, who were randomly divided to 2 groups. Randomization was stratified using a random Table number. Non-pulsatile CPB was performed on 36 patients, and pulsatile flow CPB was carried out on 36 others.

The researchers assessed preoperative renal function of each patient by examining serum creatinine, BUN levels, and urine output.

Patients with a history of renal disease, a history of liver disease and impaired liver function tests, insulin dependent diabetes mellitus (DM), morbid obesity (BMI $\geq 40$ ), redo surgery, those needing intra-aortic balloon pump, and valvular heart disease were excluded from the study. 
All of the participants were elective subjects; emergent cases were not included.

For patients taking anticoagulant medication, the drug was stopped 7 days before surgery. Patients received intramuscular morphine ( $5 \mathrm{mg}$ ) and $1 \mathrm{mg}$ of oral lorazepam 1 hour before the surgery.

The patients were taken to the operating room under standard monitoring. Vital signs and invasive blood pressure monitoring via left radial artery were recorded. Induction of anesthesia was done by fentanyl $(7 \mu \mathrm{g} / \mathrm{kg})$, diazepam (0.15 mg/kg), and atracurium ( $0.5 \mathrm{mg} / \mathrm{kg})$, if needed to the loss of eyelash reflex by continuous propofol infusion.

After intubation for maintenance, anesthesia consisted of fentanyl ( $0.07 \mu \mathrm{g} / \mathrm{kg} /$ minute), midazolam (0.5 $\mu \mathrm{g} / \mathrm{kg} /$ minute $)$ and atracurium $(0.8 \mu \mathrm{g} / \mathrm{kg} /$ minute $)$ and, if necessary, propofol (20 - $200 \mathrm{mg} /$ hour) was used. Mechanical ventilation was done and end tidal $\mathrm{CO}_{2}\left(\mathrm{ETCO}_{2}\right)$ was maintained between 35 and $40 \mathrm{mmHg}$. Central venous pressure (CVP) was inserted in the right internal jugular vein.

A urinary catheter was placed and urine output was monitored. Patient's vital signs, including blood pressure (BP) by the invasive method, pulse rate (PR), core temperature by the nasal method, oxygen saturation $\left(\mathrm{SPO}_{2}\right)$, endtidal (ET) $\mathrm{CO}_{2}$ and CVP were recorded every 5 minutes.

Arterial blood gaze (ABG) after installing the arterial line, induction of anesthesia, and immediately after the start and end of CPB was performed.

Systolic blood pressure was kept between 100 and 110 mmHg using TNG and inotrope.

After sternotomy and release of internal thoracic artery and saphenous vein, heparin was injected.

Activated clotting time (ACT) was controlled after 3 to 5 minutes of heparin injection. In case of ACT $\geq 480$ s, patients were connected to CPB pumps (type of pump was Stockert s3 with head type roller pump, and fluid prime included ringer, Voluven, heparin, bicarbonate, mannitol, trans-amine). Patients were cooled to $32^{\circ} \mathrm{C}$ to $34^{\circ} \mathrm{C}$.

During cardiopulmonary bypass, mean arterial pressure was maintained between 60 and $80 \mathrm{mmHg}$ by using noradrenaline or TNG. After completion of the flow (FULL FLOW), mechanical ventilation was stopped.

Transformation of total cross clump to partial clump was started after completing distal grafts. In the pulsatile group, ventilation resumed with $1 / 3$ of tidal volume and heart was filled by partial clumping of venous cannula accompanied with reduction of $20 \%$ to $30 \%$ in venous return.

In this way, the nonpulsatile blood flow was transformed to pulsatile blood flow by using patient's cardiac contraction. In the non-pulsatile group, continuous blood flow was continued. In both groups, temperature was in- creased to 36 to $37^{\circ} \mathrm{C}$ and ventilation was completed after the completion of proximal grafts. After confirming an acceptable situation, such as Hct $\geq 22$, normal electrolytes, and acid base status, patients were separated from СРB. Protamine sulfate was started for reverse of heparin. After completion of surgery, patients were transferred to the cardiac intensive care unit (ICU).

Weaning and extubation were performed according of standards of open heart intensive care unit (ICU-OH), such as full consciousness, hemodynamic stability, control of drainage, and chest X-ray acceptable.

Patients' laboratory tests, such as BUN and creatinine (CR) were checked at arrival of the ICU-OH, and 24 and 48 hours after admission.

Descriptive statistics such as mean, standard deviation (SD), and percentages were calculated for the presented data. The Kolmogorov-Smirnov test was used for normality of the data. Chi-square test, repeated measure, analysis of variance (ANOVA), and t test were used for determining the association between variables. The SPSS 17 software was used to analyze the data. The significance level for all tests was considered less than 0.05 .

\section{Results}

Seventy-two patients were evaluated for study participation. There was no significant difference between the 2 groups regarding gender, age, body mass index (BMI), DM, and hypertension (Table 1 ).

Table 1. Comparison Between Demographic Values (Age, Gender and BMI) and history of Diabetes Mellitus and Hypertension in the Two Groups $(\mathrm{N}=36)^{\mathrm{a}}$

\begin{tabular}{lccc}
\hline Variables & Case & Control & P Value \\
\hline Age & $61.67( \pm 8.94)$ & $62.00( \pm 7.39)$ & 0.864 \\
BMI & $30.60( \pm 2.92)$ & $30.80( \pm 3.19)$ & 0.786 \\
Gender & & & 0.405 \\
$\quad$ Male & $26(72.2)$ & $29(80.6)$ & \\
$\quad$ Female & $10(27.8)$ & $7(19.4)$ & 0.054 \\
Non-insulin depended DM & & $17(47.2)$ & \\
$\quad$ Negative & $26(72.2)$ & $19(52.8)$ & \\
$\quad$ Positive & $10(27.8)$ & & \\
Hypertension $($ HTN $)$ & & $9(25.0)$ & \\
$\quad$ Negative & $13(37.1)$ & $27(75.0)$ & \\
\hline
\end{tabular}

${ }^{\mathrm{a}}$ Values are expressed as mean ( $\pm \mathrm{SD}$ ) or Freq. (\%).

Also, there was no significant difference between the 2 groups regarding preoperative $\mathrm{CPB}$ time, cross clamp time, 
number of grafts, and systolic and diastolic blood pressure preoperative (Table 2 ).

Table 2. Comparison Between the Two Groups Related to Mean CPB and Cross Clump Times, Systolic and Diastolic Blood Pressure and Number of Grafts $(\mathrm{N}=36)^{\mathrm{a}}$

\begin{tabular}{lccc}
\hline Variables & Case & Control & PValue \\
\hline CPB Time, h: min & $1: 02( \pm 0: 22)$ & $0: 58( \pm 0: 19)$ & 0.439 \\
\hline Cross Clamp, h: min & $0: 31( \pm 0: 14)$ & $0: 33( \pm 0: 15)$ & 0.554 \\
\hline Systolic (before) & $120.61( \pm 21.48)$ & $112.18( \pm 21.76)$ & 0.110 \\
Diastolic (before) & $64.29( \pm 15.39)$ & $57.02( \pm 14.18)$ & 0.073 \\
Graft & $3.44( \pm 0.72)$ & $3.42( \pm 0.60)$ & 0.897 \\
\hline
\end{tabular}

${ }^{\mathrm{a}}$ Values are expressed as mean $( \pm \mathrm{SD})$.

Table 3 depicts creatinine mean of the study sample. The preoperative average creatinine of the participants was not significantly different between the 2 groups. However, the creatinine of the case group was significantly different on the third and second postoperative day when compared with the first day $(\mathrm{P}=0.018$ and $\mathrm{P}=0.030$, respectively).

In the control group, there was a significant difference between the first and second postoperative day $(\mathrm{P}=0.034)$.

There was a significant difference between the 2 groups in creatinine index on the first and second postoperative day $(\mathrm{P}=0.037$ and $\mathrm{P}=0.047$, respectively) (Table 3 ).

Table 4 depicts BUN mean of the study sample. The average BUN of the participants preoperatively was not significantly different between the 2 groups. The mean BUN of the case group was significantly different on the second and third postoperative day when compared with preoperative measurements, in second days after the first day postoperative and third days from second days postoperative $(\mathrm{P}$ $=0.016, \mathrm{P}=0.001, \mathrm{P}=0.043$, and $\mathrm{P}=0.001$ respectively).

The mean BUN of the control group was significantly different between the pre-operative measurement, second and third postoperative day $(\mathrm{P}=0.002, \mathrm{P}=0.003, \mathrm{P}=0.045$, and $\mathrm{P}=0.018$, respectively).

There was a significant difference between the 2 groups regarding the average BUN on the two post-operative days $(\mathrm{P}=0.027)$ (Table 4).

The mean intraoperative urine output of the case group was 2151.47 ( \pm 526.47 ) cc, and that of the control group was 2177 ( \pm 764.79 ) cc. There was no significant difference between the 2 groups regarding intraoperative urine output $(\mathrm{P}=0.376)$.

\section{Discussion}

Because renin secretion is increased due to decreased pulse pressure, non-pulsatile perfusion may effect the re- nal function more than other organs, and this could cause acute kidney failure. Besides, other physiologic outcomes of non-pulsatile blood flow constitute a release of catecholamine, vasopressin, and local tissue vasoconstrictors, resulting in vasoconstriction and raised afterload (6).

Given that even during a very short time pulsatile flow could be effective on hemodynamics and vital organ function, in this study, the researchers transferred continuous blood flow to pulsatile blood flow during proximal graft by using cardiac contraction. In this way after completion of distal graft, the surgeon turned cross clump to partial clump and after starting of heart beat they decreased the venous return by $20 \%$ to $30 \%$ using partial clumping in venous cannula. This effect on renal function was benchmarked.

In the current study, both case and control groups had significantly increased BUN and creatinine. Increased creatinine on the first and second postoperative day was significantly higher in the control group. Also, BUN increased on the second day in the control group and was significantly higher than the case group.

In a study by Poswal et al. on one hundred 35 to 65 yearold patients undergoing coronary artery bypass grafting (CABG) on CPB with normal left ventricular function, the increase in blood urea nitrogen and serum creatinine levels postoperatively at 24 and 48 hours were similar in both groups. Creatinine clearance was significantly greater in the pulsatile group on the second postoperative day. Urine output was also better in the pulsatile group compared with the non-pulsatile group (3). The current study was in agreement with these results.

In a study by Mirmohammad-Sadeghi et al. during 2010 to 2011, 589 patients were examined, who underwent CABG. They showed that patients with diabetes and older patients were more prone to acute renal failure (ARF). Also, despite increased prevalence of renal insufficiency in patients with CABG, studies have shown that in most cases, this is not a severe illness and it is directly treatable. A lower rate of participants (1.0 to $1.7 \%$ ) in several large series developed ARF, severe enough to require dialysis.

Also, in the current study increased BUN and creatinine were significant, yet none of the patients required dialysis (7).

Alghamdi and Latter in a review study on 2503 patients demonstrated that no studies determined the beneficial influence of pulsatile in decreasing the incidence of stroke or renal failure. The evidence is insufficient to recommend for or against routine pulsatile profusion for lessening the incidence of stroke or renal failure (8). 
Table 3. Comparison Between Mean Creatinine Values at Variable Times in the Two Groups $(\mathrm{N}=36)$

\begin{tabular}{lcccc}
\hline Variables & CR Before & CR Day 1 & CR Day 2 & P Value \\
\hline Case & $1.18( \pm 0.25)$ & $1.16( \pm 0.24)$ & $1.28( \pm 0.32)$ & $1.26( \pm 0.32)$ \\
Control & $1.36( \pm 0.53)$ & $1.33( \pm 0.43)$ & $1.45( \pm 0.45)$ & $1.39( \pm 0.42)$ \\
Pvalue & 0.067 & 0.037 & 0.047 & 0.115 \\
\hline
\end{tabular}

Table 4. Comparison Between Mean Blood Urea Nitrogen Values at Variable Times in the Two Groups $(\mathrm{N}=36)$

\begin{tabular}{|c|c|c|c|c|c|}
\hline Variables & BUN Before & BUN Day 1 & BUN Day 2 & BUN Day 3 & P Value \\
\hline Case & $17.72( \pm 5.81)$ & $18.33( \pm 5.58)$ & $20.19( \pm 5.57)$ & $22.36( \pm 7.23)$ & 0.001 \\
\hline Control & $20.86( \pm 9.75)$ & $22.19( \pm 11.07)$ & $24.42( \pm 9.73)$ & $25.7( \pm 10.41)$ & 0.001 \\
\hline Pvalue & 0.102 & 0.067 & 0.027 & 0.117 & - \\
\hline
\end{tabular}

\subsection{Conclusion}

Creatinine on the first and second postoperative day was significantly higher in the control group and also BUN on the second day in the control group was significantly higher than the case group. Despite the rise in BUN and creatinine in the 2 groups, there were no cases of renal failure in the patients. However, the results of this study regarding creatinine and BUN criteria were supported by the pulsatile perfusion method during the proximal graft by using a cardiac contraction in CABG surgery.

\section{References}

1. Milano AD, Dodonov M, Van Oeveren W, Onorati F, Gu YJ, Tessari M, et al. Pulsatile cardiopulmonary bypass and renal function in elderly patients undergoing aortic valve surgerydagger. Eur J Cardiothorac Surg. 2015;47(2):291-8. doi: 10.1093/ejcts/ezu136. [PubMed: 24740935] discussion 298.

2. Hökenek F, Gürsoy M, Bakuy V, Kavala AA, Demir T. , Gülcan F. , et al. An evaluation of renal functions in pulsatile and non-pulsatile cardiopulmonary bypass in the elderly. Turk J Thorac Cardiovasc Surg. 2013;21(3):610-5. doi:10.5606/tgkdc.dergisi.2013.7164.

3. Poswal P, Mehta Y, Juneja R, Khanna S, Meharwal ZS, Trehan N Comparative study of pulsatile and nonpulsatile flow during cardiopulmonary bypass. Ann Card Anaesth. 2004;7(1):44-50. [PubMed: 17827561].

4. Gourlay T, Taylor KM. Cardiopulmonary Bypass:Principles and practice. ; . Pulsatile cardiopulmonary bypass.

5. Koning NJ, Vonk AB, van Barneveld LJ, Beishuizen A, Atasever B, van den Brom CE, et al. Pulsatile flow during cardiopulmonary bypass preserves postoperative microcirculatory perfusion irrespective of systemic hemodynamics. J Appl Physiol (1985). 2012;112(10):1727-34. doi: 10.1152/japplphysiol.01191.2011. [PubMed: 22403352].

6. Sievert A, Sistino J. A meta-analysis of renal benefits to pulsatile perfusion in cardiac surgery.J Extra Corpor Technol. 2012;44(1):10-4. [PubMed: 22730858].

7. Mirmohammad-Sadeghi M, Naghiloo A, Najarzadegan MR. Evaluating the relative frequency and predicting factors of acute renal failure following coronary artery bypass grafting. ARYA Atheroscler. 2013;9(5):28792. [PubMed: 24302937].

8. Alghamdi AA, Latter DA. Pulsatile versus nonpulsatile cardiopulmonary bypass flow: an evidence-based approach. J Card Surg. 2006;21(4):347-54. doi: 10.1111/j.1540-8191.2006.00269.x. [PubMed: 16846411]. 